\title{
HIGHLY IONIZED CARBON PLASMA GENERATION BY DUAL-LASER ABLATION FOR DIAMOND-LIKE CARBON FILM GROWTH
}

\author{
S. WITANACHCHI, A. M. MIYAWA, AND P. MUKHERJEE \\ Laboratory for Advanced Materials Science and Technology (LAMSAT), Department of \\ Physics, University of South Florida, Tampa, FL 33620
}

\begin{abstract}
Carbon plasmas produced by excimer laser ablation show a low ionization yield of about 8-10\%. The coupling of a second $\mathrm{CO}_{2}$ laser pulse into the plasma in the dual-laser ablation process significantly increases the plasma temperature and the ionization. The resulting rapid expansion of the plasma gives rise to high ion kinetic energies and broader ion expansion profiles [1]. Optical emission spectroscopy and an ion probe have been used to investigate the dynamics of the carbon plasma. Single and dual-laser ablated carbon plumes have been deposited on DC-biased silicon substrates to form amorphous carbon films. The diamond-like behavior of these films was studied by Raman spectroscopy. The Raman spectra were deconvolved to gauge the effect of the density and the energy of ions on the formation of diamond-like $\mathrm{sp}^{3}$-bonded carbon (DLC) films. The advantage offered by the dual-laser process for the growth of DLC films is discussed.
\end{abstract}

\section{INTRODUCTION}

The unique properties offered by diamond, which include extreme hardness, high electrical resistivity, high dielectric strength, and high thermal conductivity, have motivated the deposition of diamond thin films for optical and electronic applications. Plasma enhanced chemical vapor deposition (CVD) is the most widely used technique for diamond film growth. Ion beam deposition, biased sputter deposition, and laser ablation are some of the alternative techniques used for the growth of diamond films[2-5]. These techniques can yield both crystalline and amorphous structures in which the properties of the films are determined by the nature of the atomic bonding. The three-fold coordinated $\left(\mathrm{sp}^{2}\right)$ bonding leads to graphitic behavior while four-fold coordinated $\left(\mathrm{sp}^{3}\right)$ bonding leads to diamond-like characteristics. The amorphous network of carbon with approximately tetrahedral local atomic configuration exhibits diamond-like properties and is thus technologically important. The amorphous diamond-like carbon (DLC) films deposited by various techniques contain an atomically mixed structure of $\mathrm{sp}^{2}$ - and $\mathrm{sp}^{3}$-bonded carbon. It has been shown that the metastable $\mathrm{sp}^{3}$ hybridization of carbon is facilitated by the thermal agitation and shock wave accompanying ion bombardment on the depositing substrate[6]. Stabilization of the metastable atomic arrangement results from the high quenching rates associated with the rapid collapse of the thermal spikes produced by the ions[7]. Therefore, atomic agitation and rapid quenching caused by energetic species during deposition is important for the growth of DLC films. Carbon ionic energies in the range of 80-100 eV have been shown to produce DLC films with a high percentage of $\mathrm{sp}^{3}$ hybridization[8]. However, much higher ionic energies cause displacement damage and surface heating which transforms the diamond-like $\mathrm{sp}^{3}$ bonds to graphitic $\mathrm{sp}^{2}$ bonds[9].

The energetic advantage of pulsed laser ablated carbon plasmas have been used by several researchers to fabricate DLC films[10,11]. Since the species energy in pulsed laser 
ablation can be controlled in the range of $1-100 \mathrm{eV}$ by the laser fluence at the target, this technique is ideally suited for the growth of DLC films. However, the percentage of ionization of a typical excimer laser ablated plume is between $4-10 \%$. The dual-laser ablation process developed in our laboratory has been shown to produce plasmas with a high percentage of ionization[12]. In addition, the high temperature of the generated plasmas leads to rapid expansion. This imparts high kinetic energy to the ions and at the same time broadens the expansion profile. In this investigation, we have used optical emission spectroscopy and a timeof-flight ion probe technique to comparatively study the dynamic expansion of the ions produced in the two laser processes. DLC films have been deposited on biased silicon substrates by both single and dual-laser processes to gauge the effect of the enhanced plume ionization on the $\mathrm{sp}^{3}$ hybridization of the DLC films. The Raman spectra of the films have been deconvolved into two distinct vibronic modes corresponding to $\mathrm{sp}^{2}$ - and $\mathrm{sp}^{3}$-bonded carbon. Based on this information, the advantages of dual-laser deposition for the growth of DLC films have been demonstrated.

\section{EXPERIMENTAL PROCEDURE}

Carbon plasma plumes have been generated in vacuum by ablating a high-density carbon target by the conventional excimer laser process and by dual-laser ablation. In the dual-laser ablation process, the excimer laser pulses of wavelength $248 \mathrm{~nm}(\mathrm{KrF})$ and $\mathrm{CO}_{2}$ laser pulses of wavelength $10.6 \mu \mathrm{m}$ are spatially overlapped on the target with an inter-pulse temporal delay of about $50 \mathrm{~ns}$. The details of this process are published elsewhere[13,14]. The experimental set up for film growth is shown in Figure 1. The excimer laser fluence at the target was about $2 \mathrm{~J} / \mathrm{cm}^{2}$

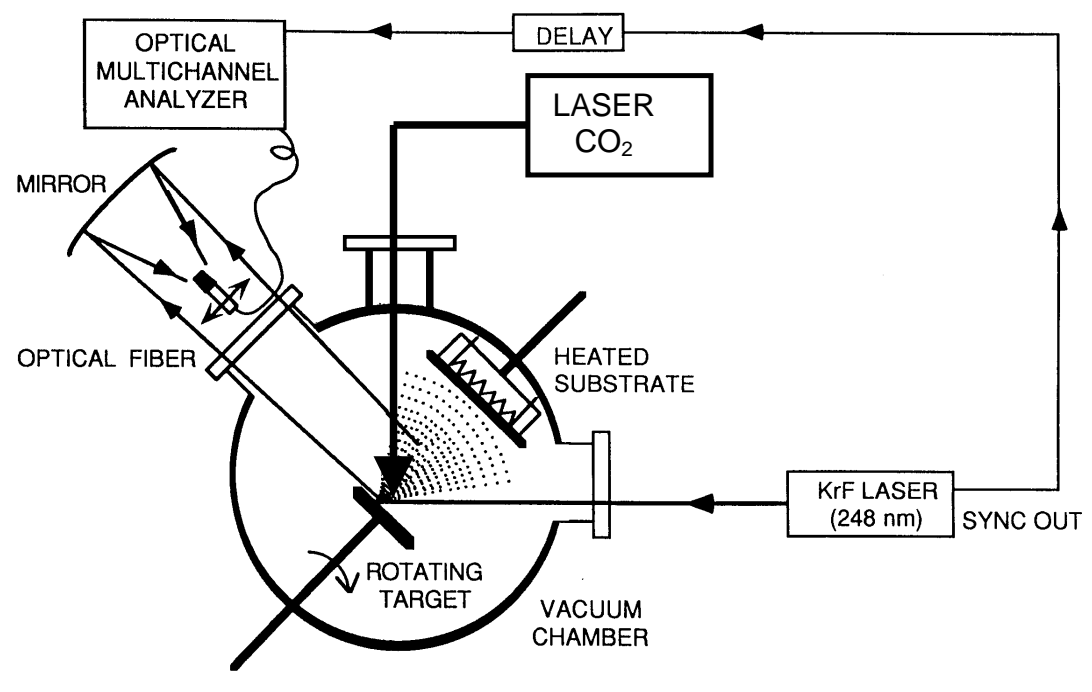

Figure 1: The dual-laser ablation system with provisions for optical emission spectroscopy of the plasma plume while the fluence of the $\mathrm{CO}_{2}$ laser was about 5 $\mathrm{J} / \mathrm{cm}^{2}$. Under optimum conditions the ablation of material is caused by only the excimer laser pulse whereas the $\mathrm{CO}_{2}$ laser pulse is absorbed into the material plasma leading to high plasma temperatures. The optical emission of the plasma plume was imaged 1:1 onto an optical fiber which was placed in the focal plane to collect emission from a point on the axis of the

plume. The fiber was connected to the spectrometer of an optical multi-channel analyzer (OMA) system, which recorded the spectra of the excited ionic and atomic species in the plume. An ion probe placed about $6 \mathrm{~cm}$ from the target also analyzed the laser-generated plasma. The probe was an insulated copper wire $1 \mathrm{~mm}$ in diameter, with only its tip exposed to the plume. It was biased at a voltage of $-10 \mathrm{~V}$. The time-of-flight ion profiles were obtained by using a $500 \mathrm{MHz}$ 
oscilloscope. The time-of-flight ion data were recorded while translating the probe in $0.5 \mathrm{~cm}$ steps in both horizontal and vertical directions with respect to the plume. Films of carbon were deposited on solvent-cleaned silicon substrates at room temperature. The bonding of the carbon atoms in the films was investigated by Raman spectroscopy using as excitation wavelength of $514 \mathrm{~nm}$.

\section{RESULTS AND DISCUSSION}

The optical emission collected from a point $5 \mathrm{~cm}$ from the target on the axis of the expanding plasma plume, for both single and dual-laser ablation is shown in Fig. 2. Most of the line emissions in single laser ablated plume are from excited atoms while high intensity emission from singly and doubly ionized carbon ions dominate the spectrum of the dual-laser ablated plume. In addition to the observed enhancement in the plume ionic content, increases in carbon ion expansion velocities were also observed for dual-laser ablated plumes. The time-of-flight ion probe intensity profiles at two points located $6 \mathrm{~cm}$ from the target are shown in Fig. 3. In

(a)

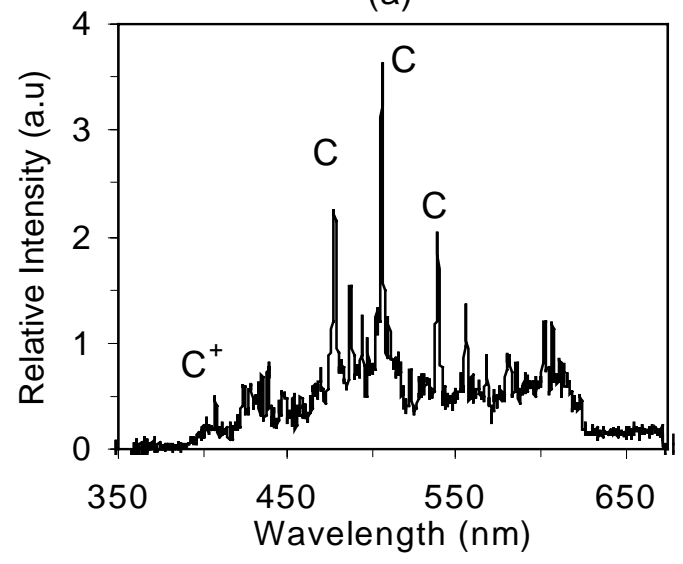

(b)

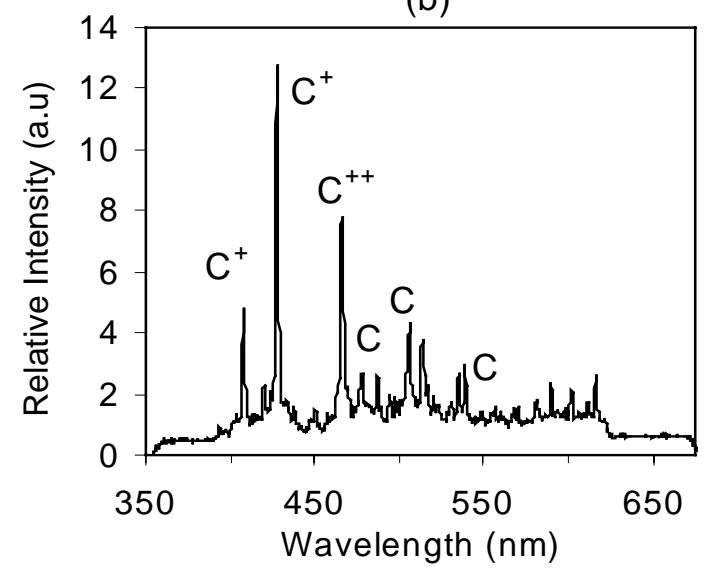

Figure 2: Optical emission from the laser-generated carbon plume $5.0 \mathrm{~cm}$ from the target for (a) single laser ablation and (b) dual laser ablation.
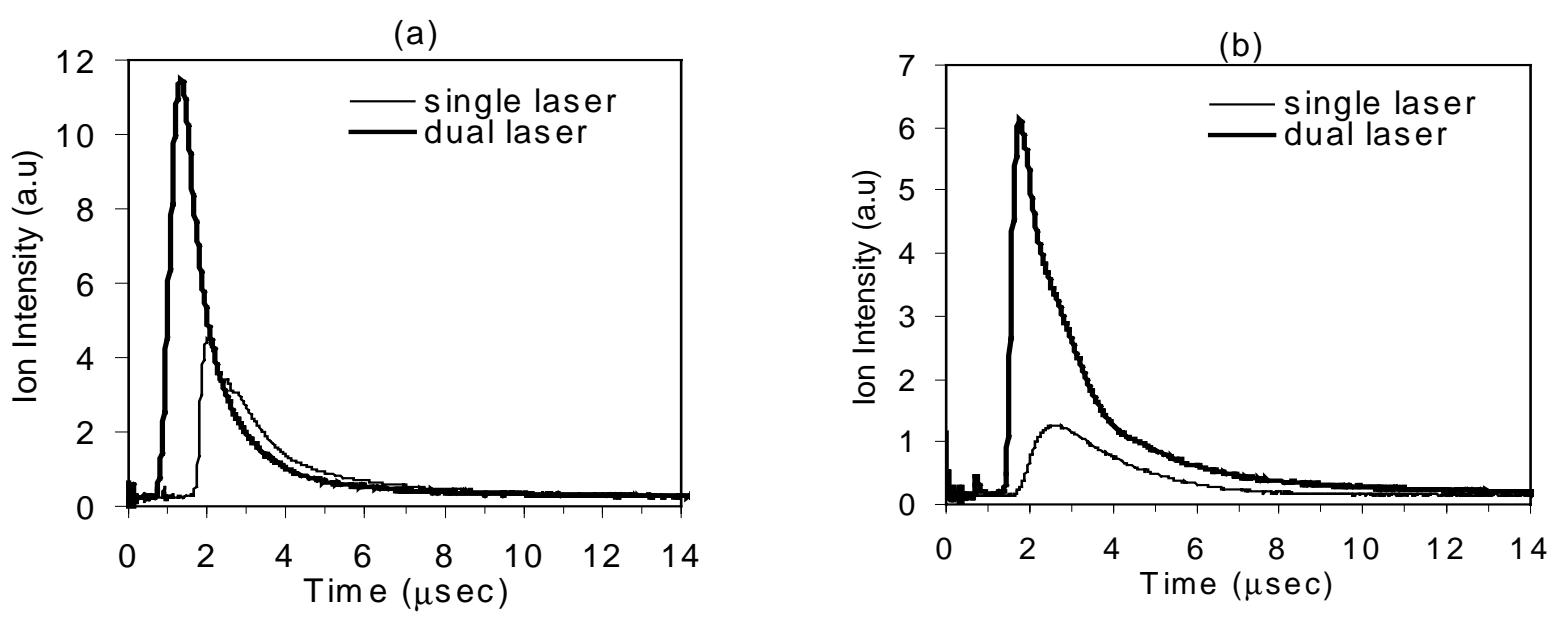

Figure 3: Time of flight ion probe signals $6.0 \mathrm{~cm}$ from the target (a) on plume axis and (b) $2.5 \mathrm{~cm}$ from the axis of the plume. 


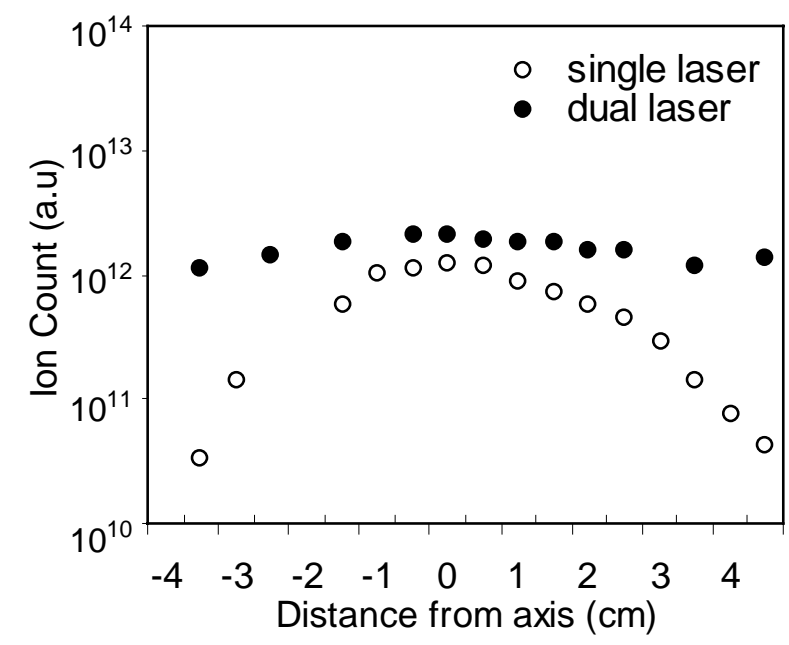

Figure 4: Ion profiles in the horizontal direction $6.0 \mathrm{~cm}$ from the target for single and dual laser ablation addition to enhancement in the ionic content of the plume, the kinetic energy of the carbon ions have undergone a significant increase in dual-laser ablation. Based on the ion profiles presented in Fig.3, the $5 \mathrm{eV}$ peak energy of the on axis carbon ions in the single laser ablated plume has been increased to $25 \mathrm{eV}$ by the dual-laser process. Similarly, the ions propagating about 23 degrees off-axis have kinetic energies of 3 $\mathrm{eV}$ and $22 \mathrm{eV}$ for single and dual laser ablated plumes, respectively. The area under the time-of-flight ion profiles obtained by scanning the horizontal and vertical directions perpendicular to the plume propagation axis were computed to derive the number of ions collected on the probe at each location. Fig. 4 shows the horizontal spatial variation of the ion distribution for single and dual-laser ablated carbon plumes. The normalized plots of these ion distributions points to a single-laser ion profile of the form $\cos ^{9} \theta$ while for dual-laser ablation this profile was of the form $\cos ^{3} \theta$. In the vertical direction, the single laser and dual-laser ion profiles were of the form $\cos ^{14} \theta$ and $\cos ^{6} \theta$, respectively. The integration of the volume under the three-dimensional ion profiles for single and dual-laser processes allows the comparison of the total ionic content in the respective plume. Our calculations showed the ionic content of the dual-laser ablated plume to be at least 5 times higher than that of the single laser ablated plumes.

To make a correlation between the ionic content and energy of the carbon ions and the quality of the DLC films, the films deposited on silicon substrates were analyzed by micro-

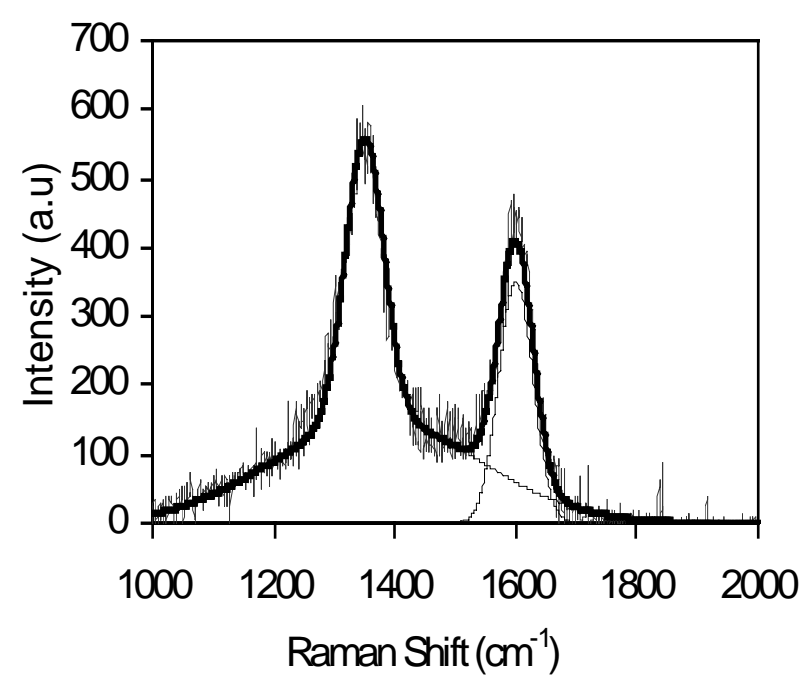

Figure 5: Raman spectrum of a carbon film deposited on a Si substrate at $500^{\circ} \mathrm{C}$.
Raman spectroscopy. It is well known that at high temperatures the $\mathrm{sp}^{2}$ hybridized graphitic phase is more stable than the $\mathrm{sp}^{3}$ hybridized diamond phase. The Raman spectrum of polycrystalline graphite formed at high temperatures consists of a "G" line at about $1600 \mathrm{~cm}^{-1}$ and a "D" line at about $1355 \mathrm{~cm}^{-1}$. It has been shown that the intensity of the $\mathrm{D}$ line is proportional to the percentage of crystalline boundaries in the sample[15]. The $\mathrm{G}$ line has been assigned to scattering of optical phonons by the graphite-like $\mathrm{sp}^{2}$ bonded carbon phase[15]. Fig. 5 shows the Raman spectrum of a carbon film deposited on silicon at a substrate temperature of $500^{\circ} \mathrm{C}$. Based on the above 
analysis, the carbon in this film is $100 \% \mathrm{sp}^{2}$-hybridized.

The Raman spectra of the amorphous carbon films deposited on silicon substrates at room temperature are shown in Fig. 6. Both spectra contain a main peak around $1530 \mathrm{~cm}^{-1}$ and a broad shoulder at around $1350 \mathrm{~cm}^{-1}$. As indicated in the Fig. 6, both spectra have been deconvolved into two components. In several experimental and theoretical investigations of amorphous DLC films, the high frequency peak ( $G$ line) has been assigned to $\mathrm{sp}^{2}$-bonded carbon clusters of fused sixfold rings while the low frequency portion has been interpreted in terms of scattering by $\mathrm{sp}^{3}$-bonded interconnecting carbon and possible contributions from the graphitelike D peak[14]. We have deconvolved the Raman spectra into three gaussian components, a component peaked at $1600 \mathrm{~cm}^{-1}$, a component peaked around $1350 \mathrm{~cm}^{-1}$ and a component peaked around $1530 \mathrm{~cm}^{-1}$. The gaussian function used was of the form

$$
I=I_{0} \exp \left(\frac{-\left(\omega-\omega_{0}\right)^{2}}{F W^{2}}\right)
$$

where, $I_{o}$ is the intensity at the peak frequency $\omega_{o}$ while $I$ is the corresponding intensity at the frequency $\omega, F W$ is a constant that corresponds to the full width at half maximum of the distribution[16]. Based on this analysis, the peak centered around $1600 \mathrm{~cm}^{-1}$ mainly represents the $\mathrm{sp}^{2}$ hybridized carbon bonds corresponding to graphite structure (Phase A), similar to the Raman spectrum of the pure graphite film (Fig. 5). The broad low frequency portion (Phase B), which was formed by combining the two components peaked around $1350 \mathrm{~cm}^{-1}$ and $1530 \mathrm{~cm}^{-1}$, corresponds to the combination of $\mathrm{sp}^{2}$ - and $\mathrm{sp}^{3}$-bonded carbon. Therefore, the ratio of the area under the curves corresponding to the two phases can be used to compare the $\mathrm{sp}^{2}-$ and $\mathrm{sp}^{3}-$ bonded carbon content of the deposited films[15]. For the single-laser deposited film, the ratio (Phase B/Phase A) is about 2.4 whereas the ratio for the dual-laser deposited film is about 7.25. This analysis clearly indicates the presence of a high percentage of $\mathrm{sp}^{3}$-bonded carbon in the dual-laser deposited films.
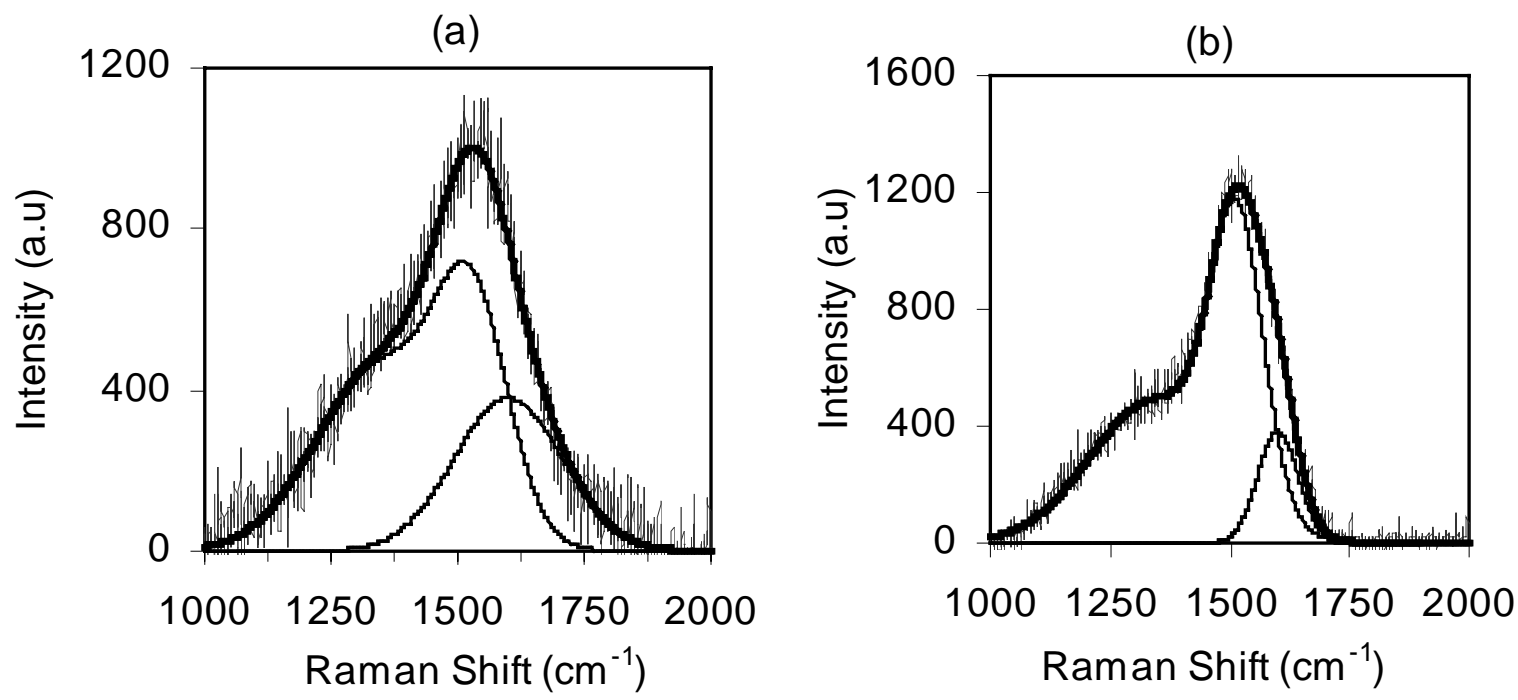

Figure 6: Raman spectra of amorphous carbon films deposited on Si substrates at room temperature by (a) single laser ablation, and (b) dual laser ablation. The solid lines indicate the two deconvoluted phases using gaussian fitting. 


\section{CONCLUSION}

In this investigation we have demonstrated that the density of carbon ions present in the dual-laser ablated plumes is more than five times higher than the ion density in single laser ablated plumes. In addition, the peak kinetic energy of the ions has been increased by a factor of six in the dual-laser ablation process. Enhanced ion flux and the ionic energy at the depositing substrate is known to promote the formation of $\mathrm{sp}^{3}$-hybridized carbon by creating localized thermal spikes. Deposition at room temperature rapidly quenches the thermal spikes preventing the transformation of diamond-like $\mathrm{sp}^{3}$-bonded carbon into graphite-like $\mathrm{sp}^{2}$-bonded carbon. In our experiments, we have shown that in comparison to single-laser ablation, the combination of high ion flux and high energy associated with the dual-laser ablation process produces DLC

films with a high content of $\mathrm{sp}^{3}$-hybridized carbon. This result, in conjunction with the ability to grow particulate free films over an extended area, clearly demonstrates the advantages offered by the dual-laser process for the growth of high-quality DLC films.

\section{ACKNOLEDGEMENTS}

This research was supported in part by the National Science Foundation (Grant No. DMI9978738) and the US Department of Energy (Grant No. DE-FG02-96ER12199).

\section{REFERENCES}

1. P Mukherjee, J. B. Cuff, and S. Witanachchi, Appl. Surface Sci. 127-129, 620 (1998).

2. F. S. Pool, Y. H. Shing, J. Appl. Phys. 68, 3276 (1984).

3. O. Matsumoto, H. Toshima, and Y. Kanzaki, Thin Solid Films 128, 341 (1985).

4. M. A. Capano, N. T. McDavitt, R. K. Singh, F. Qian, J. Vac. Sci. Technol. A14, 431 (1996).

5. A. A. Voevodin and M. S. Donley, Surface and Coating Technology 82, 199 (1996).

6. Y. Namba and T. Mori, J. Vac. Sci. Technol. A3, 319 (1985).

7. C. Weissmantel, K. Bewilogua, D. Dietrich, H. J. Erler, H. J. Hinneberg, S. Klose, W. Nowiek, and G. Reisse, Thin Solid Films 72, 19 (1980).

8. S. Ravi, P. Silva, S. Xu, B. X. Tay, H. S. Tan, and W. I. Milne, Appl. Phys. Lett. 69, 491 (1996).

9. C. Weissmantel, Thin Solid Films 58, 101 (1979).

10. V. I. Merkulov, D. H. Lowndes, G. E. Jellison, A. A. Puretzky, and D. B. Geohegan, Appl. Phys. Lett. 73, 2591 (1998).

11. A. A. Voevodin, S. J. P. Laube, S. D. Walck, J. S. Solomon, M. S. Donley, and J. S. Zabinski, J. Appl. Phys. 78, 4123 (1995).

12. S. Witanachchi, Y. Ying, A. M. Miyawa, and P. Mukherjee, Mat. Res. Soc. Symp. Proc. 485, 185 (1997).

13. S. Witanachchi, K. Ahmed, P. Sakthivel, and P. Mukherjee, Appl. Phys. Lett. 66, 1469 (1995).

14. S. Witanachchi and P. Mukherjee, J. Vac. Sci. Technol. A13, 1171 (1995).

15. J. Wagner, M. Ramsteiner, C. Wild, and P. Koidl, Phys. Rev. B 40, 1817 (1989).

16. J. Shiao, and R. W. Hoffman, Thin Solid Films 283, 145 (1996). 